\title{
LA AUTOMEDICACIÓN EN INDÍGENAS Y MESTIZOS: EL CASO DE TENEJAPA, CHIAPAS*
}

\author{
Roberto Campos, Adriana Ruiz \\ y Juana ZúÑIgA \\ (IMSS; CAS; IMSS)
}

La autoatención médica, es decir, la tentativa de resolución de un problema de salud con los propios recursos del paciente y de sus familiares, es un fenómeno que existe desde el mismo origen del hombre; esta forma de tratamiento ha consistido básicamente en el empleo de remedios - generalmente vegetales - de fácil adquisición, preparación y aplicación. Sin embargo, el consumo de medicamentos de patente o automedicación es un proceso reciente que se presenta de manera simultánea a la industrialización y comercialización de los productos farmacéuticos; en otras palabras, de la transformación de una fase artesanal (la de las "fórmulas magistrales") a otra de producción en serie, cambio que se puede ubicar inicialmente a principios de este siglo y que se incrementa notablemente en la década de los cuarentas. ${ }^{1}$

La automedicación es considerada como un primer nivel de atención asistencial presente en cualquier comunidad ya sea urbana ${ }^{2}$ o bien en zona rural, ${ }^{3}$ lo cual demuestra su amplia magnitud y la ausencia de limitantes geográficas, sin embargo ¿cuáles son los factores que permiten su existencia y reproducción? Varios son los que determinan y condicionan la automedicación, entre ellos tenemos:

Agradecemos al Lic. Eduardo Menéndez la valiosa ayuda y asesoría crítica que nos proporcionó durante la elaboración de este trabajo.

1 Salva, 1975: 236.

2 Laurell et al, 1977, 3: 408; Casillas, 1978: 95; Campos y Cano, 1979; Coe, 1973: 177; Boltanski, 1977: 19.

3 Rodríguez et al, 1979, 1: 13; Hulsof, 1979: 173; Menéndez, 1980; Silver, 1966: 471; Holland, 1963: 227; Campos, M. T. En prensa. 
1. La conversión del medicamento de un producto artesanal a una mercancía elaborada en forma seriada, con distribución amplia y con una poderosa propaganda de apoyo. Es en este punto donde las compañías farmacéuticas trasnacionales desde principios de siglo hicieron una vinculación entre el conocimiento científico y la producción material de tipo fabril, con una clara orientación lucrativa, que significó "su definitiva incorporación al proceso de producción capitalista"."

2. La transformación del médico (depositario de una ciencia y un arte con el fin de curar) en un intermediario - legalmente reconocido- entre un paciente y un medicamento o sea, un eficaz distribuidor y publicista de los fármacos de patente, con retribución económica directa o indirecta por parte de las empresas comerciales (bonificaciones, regalos, viajes, etc.). ${ }^{5}$ Menénde $z^{6}$ señala atinadamente que el personal de salud -boticarios, enfermeras y sobre todo los médicos- son los principales inductores de la automedicación en la población, dado que los fármacos son recetados no por su denominación genérica, sino por la comercial. ${ }^{7}$

3. La automedicación es un recurso menos costoso y de fácil acceso en comparación con la asistencia a un centro de atención médica que, si es privado resulta muy oneroso tanto por la consulta misma como por los medicamentos prescritos y, si es de tipo estatal es percibida como deficiente, burocrática y produce una pérdida excesiva de tiempo (espera, traslado, etc.). Este autoconsumo resulta más evidente cuando las comunidades rurales se encuentran aisladas y no disponen de accesibilidad geográfica, o económica, a un servicio médico formal. ${ }^{8}$

4. Las madres de familia, generalmente responsables de la salud familiar, son las primeras proveedoras de atención asistencial en caso de enfermedad y para ello requieren de un conoci-

4 Mercer, 1979: 110.

5 Gross, 1968: 496.

6 Menéndez, 1980: 49.

7 En las instituciones de seguridad social de nuestro país, se receta el genérico, pero el medicamento entregado al paciente conserva su nombre comercial, lo cual permite su compra y empleo a posteriori en casos patológicos similares, sin la intervención del médico.

8 Estadísticas recientes proporcionadas por la Secretaría de Salubridad y Asistencia (aparecidas en el periódico "Uno más Uno" No. 1222 del 6-0481) revelan que aproximadamente el $42 \%$ de los mexicanos no tiene acceso a la atención profesional y además existe una evidente y ostensible diferenciación de los servicios médicos establecidos dependiendo de la clase social. (Laurell 1979: 20). 
miento mínimo, elemental y útil acerca de los padecimientos y su tratamiento, estos conocimientos son adquiridos por medio de un contacto personal (llámese familiar, vecino, maestro, médico, boticario, etc.) o bien a través de los medios de comunicación masiva (libros, revistas, radio y televisión). De este modo, el conocimiento de remedios caseros y la acción de algunos medicamentos de patente se aplicará, en aquellos casos que se consideren necesarios, pudiéndose afirmar e interpretar que la automedicación es una "apropiación imitativa y simplificada, rápida e informal de la tecnología farmacéutica"日 y que, además, resulta eficaz en gran número de casos, siendo esto último fundamental para su reproducción.

En la presente investigación de campo se intenta la respuesta a las siguientes interrogantes: ¿qué características asume la automedicación en grupos étnicos diferentes? ¿Qué tan extendida es su utilización? ¿Qué tipo de medicamentos son distribuidos, consumidos y almacenados en la comunidad? Para estas preguntas se propusieron las siguientes hipótesis:

a) La automedicación se presenta en indígenas y mestizos, sin embargo es cuantitativamente mayor en el grupo con menor ingreso económico.

b) Los principales medicamentos que se distribuyen y consumen en el poblado corresponden a los sintomáticos, especialmente los analgésicos.

c) Su utilización responde a un proceso imitativo conocido en cuanto a sus efectos deseados pero desconociéndose los fenómenos farmacológicos específicos (sitio y modo de acción, biotransformación, efectos indeseables, etc.).

\section{Material y método}

Entre octubre de 1980 y febrero de 1981, se efectuaron 64 estudios a domicilio a personas residentes de las tres principales calles del pueblo de Tenejapa, de tal manera que se abarcara encuestados que vivieran en el centro de la comunidad (generalmente ladinos o mestizos) así como los que residían en la periferia de la misma (generalmente indígenas). Pasantes de la carrera de medicina que laboran en la Unidad Médica Rural (perteneciente al programa

9 Menéndez, 1980: 49. 
IMSS-COPLAMAR), ${ }^{10}$ asesorados y supervisados por el primer autor, fueron quienes aplicaron la encuesta siendo ayudados por una auxiliar enfermera con dominio del idioma tzeltal cuando en determinados casos se requería una traducción o aclaración.

Se realizaron diez estudios con el fin de probar el contenido de la encuesta y el tiempo de aplicación, como resultado se desecharon aquellas preguntas innecesarias o que estuvieran dudosas; la encuesta final incluyó cuatro secciones: en la primera se recogieron los datos generales de tipo personal del entrevistado, la segunda se relacionaba con algunos datos socio-económicos, en la tercera eran preguntas específicas acerca de la automedicación y por último se solicitaba a los encuestados que mostraran los medicamentos que en ese momento estuvieran guardados o almacenados en sus casas, interrogándoseles acerca de su adquisición, informante y propiedades farmacológicas. Durante la aplicación de la encuesta se evitó la emisión de juicios favorables o contrarios respecto a la automedicación y los remedios caseros, así como las indicaciones y efectos de los fármacos contenidos en el llamado "botiquín familiar".

Para efectos de esta investigación, la automedicación se reduce al empleo - sin prescripción médica profesional- de un medicamento llamado de "patente", ya que la automedicación en sentido amplio se refiere incluso a los remedios caseros, por ejemplo: cataplasmas, tisanas, etc.

TENEJAPA es una comunidad localizada en los Altos de Chiapas (México), con una altitud de $1,975 \mathrm{~m}$ sobre el nivel del mar, con clima húmedo-frío, se encuentra a $27 \mathrm{~km}$ de San Cristóbal de las Casas, por camino de terracería; es cabecera del municipio del mismo nombre, el cual consta de 33 comunidades llamadas parajes. Según el censo de 1970 (aun no se dispone de datos del censo realizado en 1980), existía una población de 12,930 habitantes, residiendo en $99 \mathrm{~km}^{2}$ (130 hab. $\mathrm{x} \mathrm{km}$ ); con 480 habitantes en la cabecera municipal, sin distinción étnica. Sus principales actividades económicas corresponden a la agricultura, produciendo maíz y frijol para autoconsumo, y café, caña de azúcar y cacahuate con fines comerciales. Los ingresos económicos mensuales de los indígenas son menores de 500 pesos, por lo que se ven obligados a trabajar en las zonas cafetaleras del Soconusco. Los mestizos en su mayoría se dedican

10 En mayo de 1979 se creó un convenio entre el Instituto Mexicano del Seguro Social (IMSS) y la Coordinación General del Plan Nacional de Zonas Deprimidas y Grupos Marginados (COPLAMAR) cuyos objetivos son establecer unidades médicas rurales y clínicas hospitales de campo en "zonas desatendidas". 
al comercio y en menor número a diversos oficios. La propiedad de la tierra es comunal; existen tres ejidos y "no más de cuatro propiedades privadas". ${ }^{11}$ Jueves y domingos son días de mercado; las relaciones comerciales son de tipo inter-étnico y a pesar de que los indígenas constituyen una mayoría, los ladinos ejercen una hegemonía no sólo socio-económica sino también cultural, siendo una excepción el aspecto político, ya que desde hace unos 40 años el ayuntamiento municipal está dirigido por indigenas, exceptuanda al secretario que es mestizo y cuya función es asesorar y orientar al presidente municipal. Además existen representantes de cada paraje y autoridades religiosas encargadas de preservar las ceremonias rituales.

Los criterios con los que el indígena se identifica a sí mismo son: el hablar tzeltal, vivir en los parajes en casas de barro con techo de zacate, vestir chamarro, no tener dinero y no hablar (o muy poco) el español; en tanto que el ladino se identifica por hablar el "castilla", tener dinero, vestir ropa "occidental" y vivir en la cabecera municipal en casas de cemento. ${ }^{12}$

Dadas las condiciones de extrema pobreza, las principales causas de morbi-mortalidad corresponden a las gastroenteritis, infecciones respiratorias agudas, parasitosis intestinales, tuberculosis y cirrosis hepática (por cierto, padecimientos típicos de la explotación dentro del sistema capitalista). El municipio dispone de un Centro de Salud (de la Secretaría de Salubridad y Asistencia) con un médico pasante en servicio social, cuatro Unidades Médicas Rurales del IMSSCOPLAMAR (3 de ellas localizadas en parajes), un Centro Promocional de la Salud a cargo de un enfermero, con uria farmacia anexa y, por supuesto, las tiendas que expenden casi todo tipo de fármacos. En caso necesario los enfermos pueden asistir o ser llevados a la ciudad de San Cristóbal de las Casas, donde hay diversas instituciones gubernamentales, médicos privados y un gran número de farmacias. Además dentro del municipio se encuentra todo tipo de practicantes de la denominada medicina tradicional: parteras, curanderos, etc.

\section{Resultados}

Datos generales. De las 64 encuestas realizadas, 30 se hicieron a indígenas, 19 hombres y 11 mujeres cuyas edades variaron entre los

11 Sánchez, 1980.

12 Vázquez, 1978: 220. 
19 y 75 años, la mayoría eran casados. Con respecto a la escolaridad hubo 21 analfabetas $(70 \%), 7$ con primaria incompleta $(23.3 \%)$, uno con primaria completa y otro con estudios de normal para maestros (3.3\% respectivamente) (ver cuadro No. 1 ). Sólo 4 eran originarios de la cabecera municipal; el resto con lugar de nacimiento en los diversos parajes del municipio, predominando La Cañada, distante $5 \mathrm{~km}$ de Tenejapa. Por supuesto todos hablan tzeltal; no se investigó cuántos hablaban el español. Predominó la religión católica $(73.3 \%)$, siguiendo la protestante evangélica (20\%); dos encuestados negaron filiación religiosa.

Con respecto a los mestizos, se obtuvieron 34 encuestas, $22 \mathrm{mu}-$ jeres y 12 hombres, cuyas edades fluctuaron entre 17 y 80 años, hubo 25 casados, 7 viudos y 2 solteros. En relación a la escolaridad, se observó que 16 tenían primaria incompleta (47\%), 10 en forma completa $(29.4 \%), 4$ analfabetas $(11.7 \%)$, uno con secundaria incompleta, 2 que la finalizaron y uno con estudios de magisterio. La gran mayoría (82\%) nació en Tenejapa, el resto en otros municipios colindantes: Chenalhó, Huixtán y San Cristóbal de las Casas. Además de hablar el español, 25 dominan el tzeltal (74\%) y uno el tzotzil. Todos se manifestaron como católicos.

Datos socio-económicos. El $83.3 \%$ de los padres de familia indígenas se dedican a la agricultura, un $10 \%$ realiza labores de pequeño comercio y el resto son empleados. El salario expresado en la encuesta varió entre los 70 pesos y 12,000 pesos mensuales, este último correspondiendo a un maestro y su esposa, también maestra; el promedio general fue de 1,051 pesos mensuales (ver cuadro No. 2). Debe aclararse que el $26 \%$ reconoció su traslado a las fincas cafetaleras. El tipo de familia predominante fue el de tipo nuclear (66\%) y el resto de tipo extenso. El promedio de integrantes del núcleo familiar correspondió a 5.4, con un mínimo de 2 y un máximo de 13 .

Por otra parte, el $26 \%$ de los ladinos se dedican al comercio (tiendas y tendajones), un porcentaje similar tiene empleo remunerado que procede de organismos gubernamentales; un $17.6 \%$ se dedica a labores agrícolas y el resto a oficios diversos y trabajo doméstico. Entre los mestizos, el ingreso mensual menor fue de 240 pesos y el mayor de 9,500 pesos, con un promedio de 3,500. La familia nuclear predominó sobre la extensa $(68 \%$ y $32 \%$ respectivamente), encontrándose un 4.5 como promedio de miembros familiares, con un mínimo de 1 y un máximo de 8.

Consumo de medicamentos. El $87 \%$ de los indígenas (ver cuadro No. 4), aceptó el consumo de fármacos de patente sin prescripción 
médica, siendo los principales motivos de empleo aquellos casos en los que el síntoma dolor es predominante (dolor de cabeza, de estómago, etc.) así como ante la presencia de diarrea y tos. De las 30 familias indígenas encuestadas, más de la mitad $(53 \%)$ refirieron que la utilización de dichos medicamentos NO constituía un peligro en su aplicación: "porque si el médico lo receta, creemos que no son peligrosos", "son buenos"; en tanto que el $36.6 \%$ afirmó el conocimiento de efectos negativos: "pueden caer mal al cuerpo", "tal vez sea fuerte la reacción", "porque la gente se puede intoxicar y morir", etc. Cinco de ellos no pudieron explicar el porqué.

Comparando la efectividad de los productos farmacéuticos en relación a los remedios caseros, el $87 \%$ de los indígenas creían que los primeros eran más eficaces: "curan más rápido", "son más fuertes", "quitan el dolor", "es más bueno" y "pueden más que la hierba". Un 10\% opinó que eran iguales: "las hierbas también mejoran", "todo cura, algunas veces los remedios curan más que las medicinas" y "lo mismo hace el remedio que la medicina". Los medicamentos encontrados en el hogar de 22 familias correspondieron a los siguientes genéricos: analgésicos (37), antibióticos (13), antiparasitarios (8), para vías respiratorias (3), vitamínicos (4) y varios (5) (ver cuadro No. 3), generalmente expedidos sin receta médica en las tiendas del poblado y en menor frecuencia por boticario y enfermera; no se encontraron fármacos recetados por el médico de la Unidad Médica Rural. Llamó la atención que un medicamento antipalúdico $\left(\right.$ Aralen $\left.^{R}\right)$ era utilizado para combatir el dolor de cabeza, habiendo sido recomendado por un tendero.

Con respecto a los mestizos, un $83 \%$ aceptó la autoprescripción en casos considerados como "pasaderos", "no graves", "simples" y "sencillos", principalmente para cefaleas, dolor estomacal, catarros y diarreas. El 68 tenía conocimiento de efectos nocivos de algunos productos: "las inyecciones pueden estar pasadas", "los anticonceptivos dan muchos nervios y las mujeres pueden quedar locas", "por no saber las dosis", "los antibióticos pueden producir alergias", "la penicilina mata los glóbulos rojos" y "porque pueden ser para otra cosa". Un 33\% negó tal peligrosidad: "porque si fueran peligrosas no se usaran", "si un doctor lo receta no creo que haga daño" y "porque curan".

Un $85 \%$ de los ladinos afirmó que los medicamentos eran más efectivos que los remedios caseros: "porque van directas", "porque sana más rápido al enfermo", "más preparadas" y "tienen patentidad". Tres de los encuestados refirieron que el efecto en ambas 
formas de tratamiento era similar: "las dos nos han aliviado" y "hacen igual". Sólo un encuestado manifestó que los remedios caseros eran superiores a los fármacos aduciendo que "hacen menos daños". Los medicamentos guardados en 23 hogares correspondieron a los siguientes genéricos: analgésicos (18), para vías respiratorias (16), varios (16), antiparasitarios (8), vitamínicos (5) y antibióticos (4). Los principales distribuidores fueron, en orden decreciente: boticario, tendero, médico de Unidad Médica Rural y por último la enfermera. Se observó además una extensa variedad de nombres comerciales y la presencia de productos relacionados con padecimientos crónicos degenerativos y para casos de hipertensión arterial.

En los cuadros 4 y 5, al correlacionarse la automedicación con el grado de educación formal así como con el ingreso económico familiar, y excluyendo la diferencia étnica, se observa una mayor frecuencia de automedicación en individuos de baja escolaridad y en sectores extremos de ingreso económico. Por último, a través de la observación realizada en las tiendas de la comunidad, encontramos que los medicamentos expuestos a la venta corresponden en su mayoría a los analgésicos en sus diversas presentaciones, siendo la compra por unidad y no por envase completo.

\section{Comentarios}

Como se habrá observado, existe una evidente diferenciación entre ambos grupos, los indígenas son en su mayor parte campesinos con un elevado grado de analfabetismo y un reducido ingreso salarial, comparados con el grupo mestizo con el cual conviven y que en su mayoría labora en actividades comerciales y de servicios; sin embargo, el factor cultural étnico es secundario e incluso insuficiente para explicar dicha situación desigual. Creemos que lo que determina y condiciona este tipo de estratificación es la manera en que cada agrupación se articula al modo de producción dominante, es decir, el capitalista, sistema que genera y reproduce - a través de las instancias socio-económicas y superestructurales o ideológicaslas actuales condiciones de vida de unos y otros, así como su mutua interacción, considerando que los mestizos forman parte de la burguesía comercial rural ${ }^{13}$ y los indígenas constituyen un sector social

13 García de León, 1978: 135, refiere que "la burguesía comercial rural... es la que directamente se relaciona con las comunidades y que a nivel primario constituye el principal enemigo reconocible para los sectores dominados". 
integrado por campesinos pobres pequeño-productores, en el que algunos, por el déficit en su producción agrícola, tienden a proletarizarse al vender su fuerza de trabajo en forma asalariada en regiones clave de la agricultura capitalista, cuya producción es de exportación.

Refiriéndonos ya específicamente al tema de esta investigación, se deduce por los datos obtenidos que la automedicación es un fenómeno ampliamente difundido sin diferencia cuantitativa demostrable al considerar la variable étnica, encontrándose algunas particularidades relacionadas a patrones de consumo, por ejemplo: los indígenas acuden con mayor frecuencia a solicitar información y compra de medicamentos con el tendero que con otro tipo de posibles proveedores (boticarios, médico, etc.), que emplean más los analgésicos y los antibióticos. Los mestizos acuden más con el boticario y el personal médico, utilizando una mayor diversidad de fármacos incluyendo aquellos que terapéuticamente tienen valor para enfermedades crónicas, lo cual sugiere la existencia de una patología diferencial en la misma comunidad, dato que no ha sido captado y analizado en las estadísticas de morbi-mortalidad que poseen las instituciones sanitarias que laboran en Tenejapa.

Por otra parte, se confirma que la automedicación es un proceso imitativo, simplificado y complementario de una técnica curativa, teniéndose una imagen del medicamento en función de la rápida y eficaz resolución de algunos síntomas o padecimientos catalogados como leves, aun por encima de los llamados remedios caseros, no asimilándose (o haciéndolo en forma fragmentaria y distorsionada) el discurso médico "oficial" acerca de las características farmacológicas del producto consumido (indicaciones, contraindicaciones, efectos colaterales, etc.), lo cual conlleva peligros que los indígenas en su mayoría desconocen; desconocimiento que por otra parte se encuentra en otros sectores sociales.

Es oportuno mencionar que la automedicación es y ha sido atacada en forma constante y violenta por parte de algunos médicos, siendo frecuente y cotidiano ver y escuchar comentarios acerca de los efectos negativos y perjudiciales que produce esta práctica, olvidándose de que en la praxis médica, tal y como se ejerce en los países capitalistas dependientes, como el nuestro, esta automedicación responde a la lógica del lucro y la ganancia ilimitada, y que son precisamente las empresas farmacéuticas las primeras interesadas en su desarrollo y crecimiento. Éstas utilizan eficientes estrategias de penetración comercial tanto en zonas urbanas como rurales, con la aparente 
intención de "atacar la enfermedad y el dolor humano", aunque en realidad sus fines son puramente mercantiles. A esto hay que añadir - como ya se mencionó en la introducción del presente trabajo- la ausencia de una infraestructura sanitaria (agua potable, drenaje, etc.) en muchas localidades rurales, la falta de atención médica, la ínfima calidad del servicio médico en las poblaciones en que sí se encuentra, etc.

Resolver el problema que plantea la automedicación (o sea la presencia de efectos nocivos y colaterales), no depende ciertamente de la prohibición generalizada de dicha práctica (lo cual es utópico), pero sí modificaciones en su orientación en cuanto exista un cambio de las estructuras socio-económicas que garantice:

- La apropiación social o colectiva de los medios de producción y consecuente nacionalización de la industria químico-farmacéutica.

- Desaparición de la medicina privada y creación de un servicio nacional de salud que permita el ejercicio de actividades médico sanitaristas igualitarias, de fácil acceso a todos los mexicanos y de óptima calidad (características que son prácticamente imposibles dentro de la estructura capitalista).

- La creación y constante revisión de un cuadro básico de medicamentos que incluya aquellas presentaciones que verdaderamente sean útiles para la curación y control de las enfermedades más comunes en nuestro medio, eliminando las marcas comerciales. ${ }^{14}$

- La creación de un sistema nacional de distribución y comercialización que dependa del Estado y que susbtituya la actual organización privada.

- Un estrecho y eficaz control estatal de los medicamentos considerados como de uso muy delicado (drogas, antibióticos, etc.).

- Y por último, la difusión popular o vulgarización a través de los medios masivos de comunicación, del conocimiento médico farmacológico de aquellos productos sencillos, como los analgésicos, antitusígenos, etc., que ocasionalmente pueden provocar algún daño corporal aún a dosis normales.

Coyoacán, agosto de 1981.

14 De hecho en la actualidad existe en México un cuadro básico que sólo es empleado por las instituciones estatales de salud. 


\section{OBRAS CITADAS}

Boltanski, Luc

1977 Descubrimiento de la enfermedad. Ed. Ciencia Nueva. Buenos Aires.

Campos, María Teresa

en prensa "El sistema médico Tojolabal", en Los Legítimos hombres. Aproximación antropológica al grupo tojolabal, vol. 3, Edición de Mario Humberto Ruz, Centro de Estudios Mayas, UNAM, México.

Campos Navarro, Roberto y Joel Gano Téllez

1979 Medicina popular-tradicional y automedicación en familias asistentes a la clinica 25 del IMSS, Tesis de especialización en

Casillas, Leticia Medicina familiar. Mimeografiado. México, D. F.

1978 "El uso de recursos médicos en el hogar. Estudio de familias urbanas y suburbanas de la ciudad de México" en Estudios

Coe, Rodney sobre Etnobotánica y Antropología Médica, III, México, D. F.

1973 Sociología de la Medicina. Ed. Alianza. Madrid.

García de León, Antonio

1978 "Sobre estructura de clases e ideología en regiones indígenas en Chiapas" en Economía campesina y capitalismo dependiente.

Gross, Martin UNAM, México.

How AND, Wuram

1968 Los docto

1963 Medicina maya en los Altos de Chiapas. Un estudio del cambio sociocultural. Instituto Nacional Indigenista, Serie de Antro-

HulshoF, JoséE pología Social núm. 2, México, D. F.

1979 "El consumo de medicamentos en el Altiplano de Bolivia y la Sierra Central de Perú" en Foro sobre empresas multinacionales y transferencia de tecnología en el ramo de la industria químico farmacéutica, México, D. F.

laureli, Asa Cristina, Arcelia Blando y Alfredo Heredia

1977 El desarrollo urbano y los patrones de consumo de productos farmacéuticos. Una encuesta en diferentes grupos sociales de México, D. F., Salud Pública de México 19 (3).

1979 Introducción en Clases Sociales y Enfermedad. Introducción a une epidemiología diferencial. Ed. Nueva Imagen, México, D. F.

MEnÉndez, Eduardo

1980 Clases subalternas y el problema de la medicina denominada "tradicional". Cuadernos de la Casa Chata núm. 32, México, D. F.

Mercer, Hugo

1979 "La prescripción de medicamentos" en Foro sobre empresas multinacionales y transferencia de tecnología en el ramo de la industria químico farmacéutica, México, D. F.

Rodríguez, José et al.

1979 Tratamientos no médicos de la enfermedad en el medio rural mexicano. Estudio en los usuarios de un centro de salud $C$ en el estado de Morelos, Salud Pública de México 21 (I). 
SAzva, M.

1975

"Técnicas farmacoterapéuticas" en Historia Universal de la Medicina, Ed. Salvat, México, D. F.

Ś́NCHEZ, HÉCTOR

1980 Estudio de comunidad en Tenejapa, Chiapas. Mimeografiado. SILVER, DANIEL

1966 "Enfermedad y curación en Zinacantán" en Los Zinacantecos, Vírguez, Luis Editor Evon Vogt, INI, México.

1978 "Sistema de mercado y relaciones interétnicas: estudio de Tenejapa, Chiapas" en Economía campesina y capitalismo dependiente. UNAM, México. 


\section{CUADRO L -ESCOLARIDAD COMPARADA -}

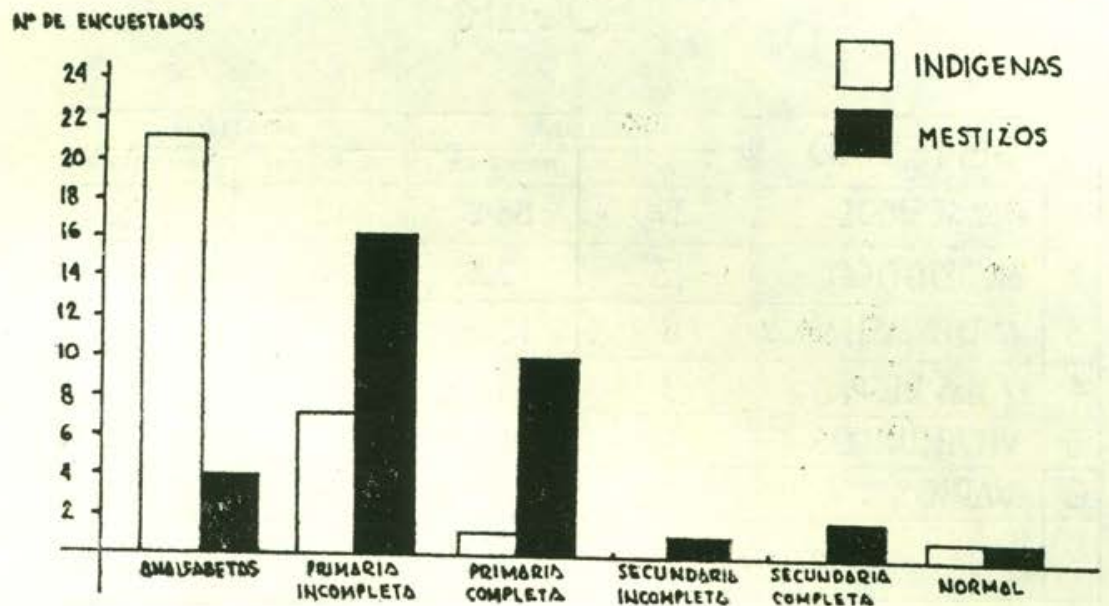

\section{CUDDRO 2 - INGRESO ECONOMICO MENSUAL COMPARADO -}

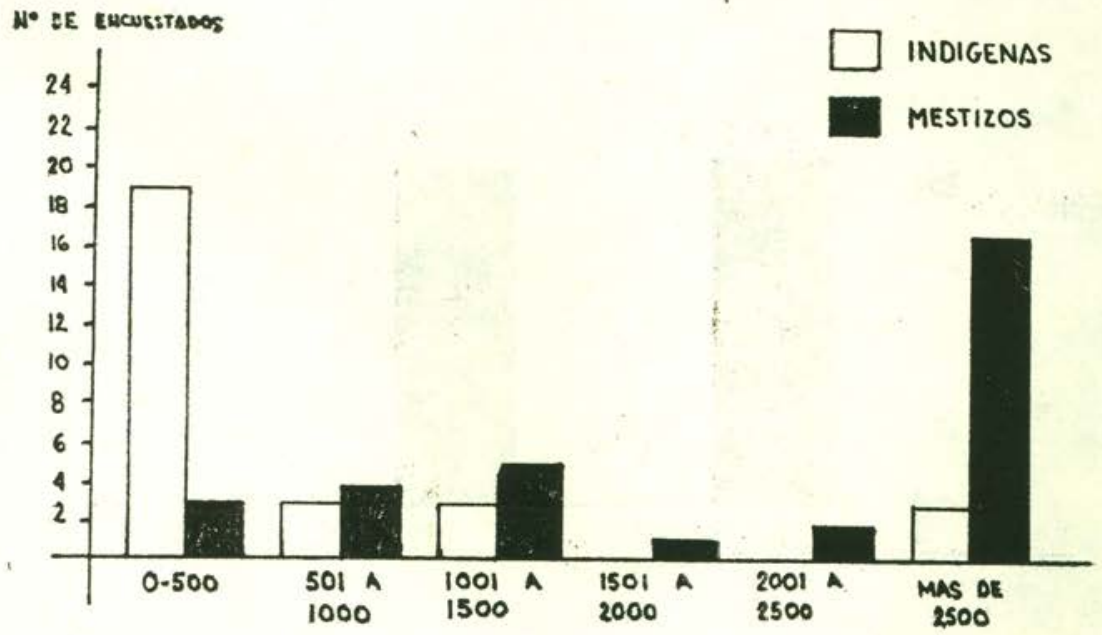




\section{CUADRO 3 -MEDICAMENTOS ALMACENADOS EN EL HOGAR}

\begin{tabular}{|c|l||c|c||c|c|}
\hline \multicolumn{2}{|c|}{ - GENERICO } & \multicolumn{2}{c||}{ INDIGENAS } & \multicolumn{2}{c|}{ MESTIZOS } \\
\cline { 2 - 5 } & N. & PORCENTASE & N. & PORCENTASE \\
\hline 2 & ANSLGESICOS & 37 & $53 \%$ & 18 & $27 \%$ \\
\hline 3 & SNTIBIOTICOS & 13 & $19 \%$ & 4 & $6 \%$ \\
\hline 4 & P/ VIISS RESP. & 3 & $4 \%$ & 16 & $24 \%$ \\
\hline 5 & VITAMINICOS & 4 & $6 \%$ & 5 & $7 \%$ \\
\hline 6 & VARIOS & 5 & $7 \%$ & 16 & $24 \%$ \\
\hline & TOTAL & 70 & $100 \%$ & 67 & $100 \%$ \\
\hline
\end{tabular}

CUADRO 4 - AUTOMEDICACION GRUPO ETNICO-

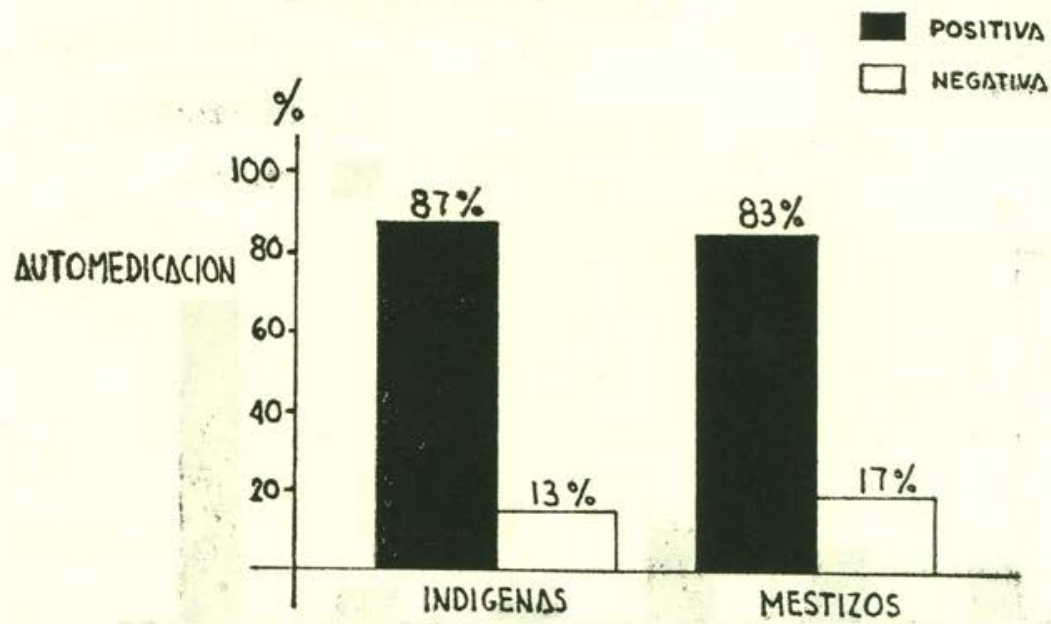




\section{CUADRO 5 AUTOMEDICACION ESCOLARIDAD}

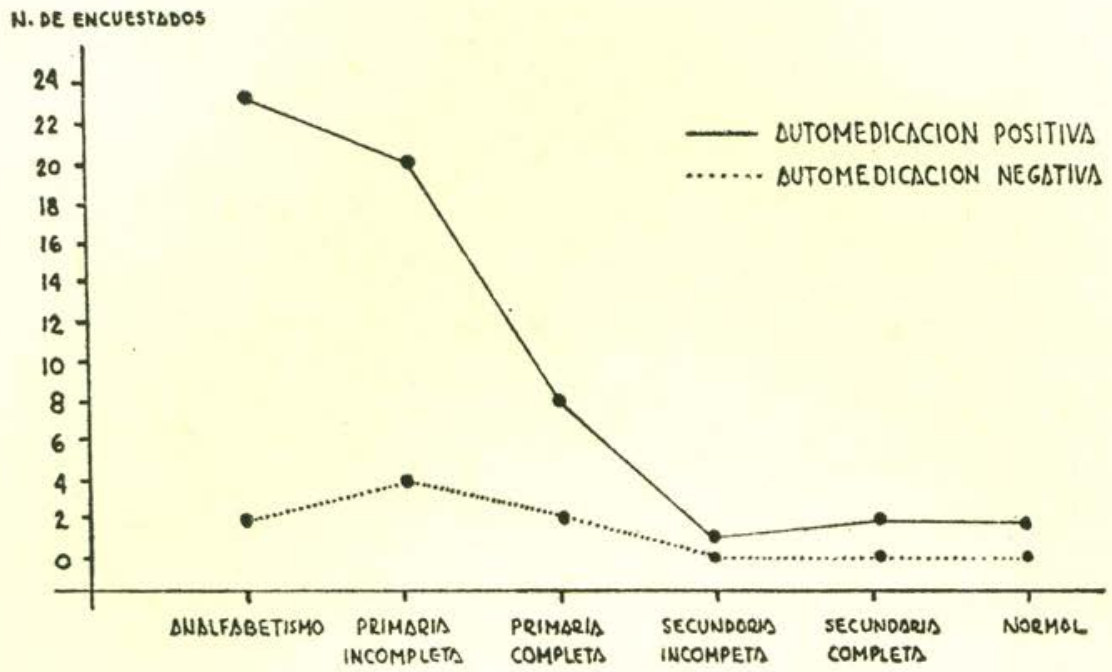

CUADRO 6 AUTOMEDICACION E INGRESO ECONOMICO

N. DE ENCUESTADOS

- AUTOMEDICACION POSITIVA

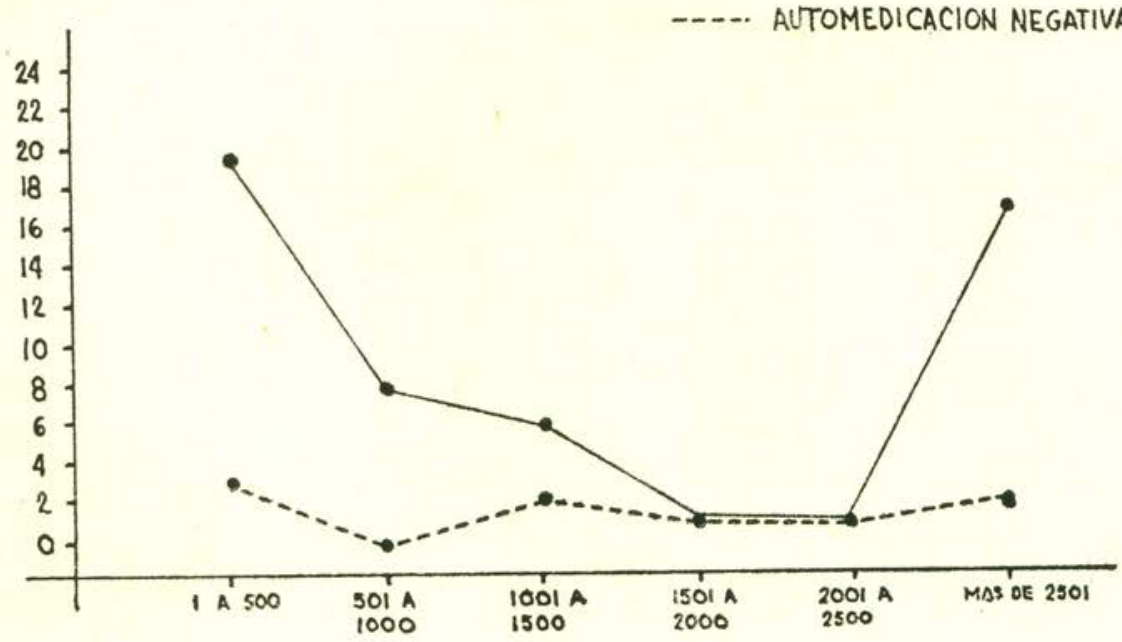

